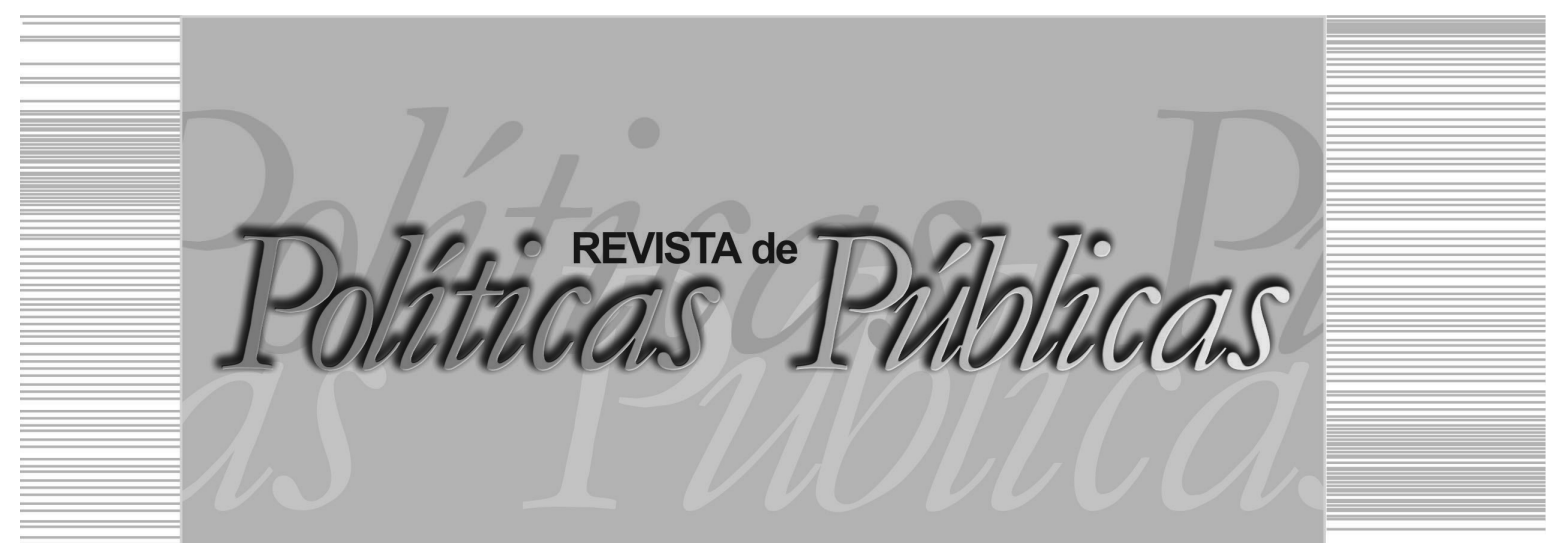

\title{
ATENÇÃO PRIMÁRIA À SAÚDE NA AGENDA PÚBLICA BRASILEIRA: dilemas entre focalização e universalidade
}

\author{
Mônica de Castro Maia Senna ${ }^{l}$ \\ Universidade Federal Fluminense (UFF) \\ Marta Alves Santos ${ }^{2}$ \\ Prefeitura de Niterói/ABEU Centro Universitário (UNIABEU)
}

\section{Resumo}

O presente artigo traz uma breve reflexão sobre os desafios postos à Atenção Primária à Saúde (APS) no contexto brasileiro. Para tanto, apresenta a construção do conceito de APS no âmbito internacional e nacional e destaca sua trajetória histórica no país. Examina algumas inflexões trazidas pela centralidade da APS na agenda pública nacional e, por fim, indica alguns desafios e possibilidades que se apresentam à Atenção Primária num cenário de impasses e limites que se destacam entre focalização e universalidade na implementação da APS no país.

Palavras-chaves: Atenção Primária à Saúde, universalidade, política de saúde.

\footnotetext{
1 Assistente Social, Doutora em Ciências - Saúde Pública - FIOCRUZ, Professora Associada da Universidade Federal Fluminense (UFF). E-mail: monica.senna20@gmail. com / Universidade Federal Fluminense (UFF): Reitoria da UFF, Rua Miguel de Frias, 9, Icaraí - Niterói - RJ. CEP: 24220-900

2 Assistente Social, Doutora em Política Social pela Universidade Federal Fluminense (UFF), Assistente Social da Prefeitura de Niterói - RJ e da ABEU Centro Universitário / Prefeitura de Niterói: Rua Visconde de Sepetiba, 987, Centro - Niterói - RJ. CEP: 24.020206; ABEU Centro Universitário - UNIABEU: Rua Itaiara, 301 - Centro - Belford Roxo - RJ. CEP: 26113-400
} 


\title{
PRIMARY HEALTH CARE AT THE BRAZILIAN PUBLIC
}

AGENDA: dilemmas between focalization and universality

\begin{abstract}
This paper provides a brief reflection on the challenges posed to Primary Health Care (PHC) in Brazil. It presents the construction of the PHC concept in international and national contexts and highlights its trajectory Brazil. The text examines some inflections brought by the centrality of APS in the national public agenda. Finally, it indicates challenges and possibilities for Primary Health Care into the trade off between targeting and universality in Brazilian case. Key words: Primary Health Care, universality, health policy.
\end{abstract}

\section{INTRODUÇÃO}

O reconhecimento de que a saúde é um direito universal constitui um processo bastante recente na sociedade brasileira. Até a Constituição Federal (CF) de 1988, predominava no país um modelo dual de atenção à saúde, combinando ações preventivas, de caráter coletivo e massivo, dirigidas ao conjunto da população e à assistência médica previdenciária, exclusiva dos segurados da Previdência Social. Essa última, hegemônica, orientava-se por uma concepção médico-curativa hospitalocêntrica, centrada no paradigma flexneriano, cujos fundamentos repousam em uma abordagem mecanicista do processo saúde-doença, no reducionismo da causalidade a aspectos biológicos e no foco da atenção na doença e no indivíduo. (BAPTISTA, 2005).

É em meio à intensa mobilização social que marcou o processo de redemocratização da sociedade brasileira entre o final dos anos 1970 e os 1980, que a noção de direito universal à saúde se fortalece. Impulsionada pela luta do chamado movimento sanitário, a defesa da atenção à saúde como um direito de todos e dever do Estado se associou à perspectiva de resgate da dívida social brasileira e ampliação da cidadania.

O texto constitucional incorporou parte dessas aspirações e, de forma inédita no país, inscreveu o direito à saúde no rol da seguridade social brasileira, com um atraso de quarenta anos em relação à concepção inglesa que a inspirou. (BOSCHETTI, 2003). Ao instituir o Sistema Único de Saúde (SUS), a CF de 1988 (e posteriormente a Lei Orgânica da Saúde) definiu a universalidade, a descentralização e a participação social como princípios norteadores do novo sistema, 
ATENÇÃO PRIMÁRIA À SAÚDE NA AGENDA PÚBLICA BRASILEIRA:

dilemas entre focalização e universalidade

rompendo, do ponto de vista normativo-legal e com forte apelo simbólico, com critérios de acesso amparados na contribuição prévia ou associados à miséria.

Sem dúvidas, a institucionalização do SUS expandiu a oferta de serviços de saúde em todos os níveis do sistema e ampliou a cobertura das ações sanitárias, englobando um conjunto de intervenções no âmbito da prevenção, promoção, recuperação e reabilitação, agora dirigidas a toda a população. Os números referentes ao SUS são surpreendentes: de acordo com informações disponíveis na página virtual do Ministério da Saúde (MS), em 2014 o SUS realizou 4,1 bilhões de procedimentos ambulatoriais, 1,4 bilhões de consultas médicas, 11,5 milhões de internações, 19 milhões de procedimentos oncológicos, mais de 23 mil transplantes de órgãos e tecidos, além de avançados programas de saúde, dentre eles o de controle e tratamento da AIDS. (POPULAÇÃO..., 2015).

Apesar de sua robustez, a condução do SUS tem se defrontado com diversos desafios, associados, em grande parte, às características estruturais da sociedade brasileira (com destaque para a profunda desigualdade social que marca a história do país) e à adoção de medidas contencionistas pelos sucessivos governos que se seguiram à promulgação do texto constitucional, o que tende a comprometer a própria noção de direito à saúde nele inscrita.

De fato, desde os anos 1990, mais especificamente a partir da segunda metade da década, o processo de implementação do SUS tem sido marcado pela tensão entre sua consolidação como sistema público e universal e o privilegiamento de ações centradas na pobreza, com severas restrições orçamentárias e corte nos gastos públicos. Dentro desse contexto, a denominada Atenção Primária à Saúde (APS) ganha centralidade na agenda governamental, com a instituição da Estratégia Saúde da Família (ESF). Inicialmente concebida como programa de expansão de cobertura para áreas rurais e mais empobrecidas do país, aos poucos a ESF passa a figurar como estratégia central de reorganização do sistema de serviços de saúde no país.

A prioridade conferida à ESF tem suscitado um intenso debate em torno de suas repercussões no alcance do direito universal à saúde, demonstrando que não existe consenso em torno da questão. Alguns estudiosos têm chamado atenção para o fato de que a ESF tem caminhado em direção ao fortalecimento de um padrão de 
intervenção estatal focalizado nos mais pobres, com a perspectiva de racionalização de gastos em detrimento de políticas universais. (FRANCO; MERHY, 2000). Outros autores enfatizam os avanços promovidos pela ESF em direção à reconfiguração do modelo de atenção, um dos princípios contidos na CF de 1988 e na Lei Orgânica da Saúde. (MENDONÇA, 2009).

O presente artigo pretende contribuir nesse debate, trazendo alguns elementos identificados no exame do processo de consolidação da ESF em anos recentes, vis-à-vis sua articulação aos princípios constitucionais do SUS. Sob esse prisma, são apresentados o conceito e as diferentes interpretações sobre a APS tanto no âmbito internacional, quanto no nacional. Em seguida, apresenta-se a trajetória recente da Atenção Primária no Brasil com a centralidade conferida à ESF, considerando as dinâmicas tecidas em torno das principais inovações e dos desafios postos para a referida política na atual conjuntura.

\section{ATENÇÃO PRIMÁRIA À SAÚDE: conceitos e}

interpretações no cenário internacional

O debate em torno da APS ganha expressão a partir de meados da década de 1970, com destaque para a Conferência Internacional sobre Cuidados Primários de Saúde, realizada em Alma-Ata (atual Cazaquistão) no ano de 1978. Tal debate foi fortemente impulsionado por questionamentos ao modelo então hegemônico de organização da atenção à saúde, em particular a seu caráter médico-centrado, hospitalocêntrico, intervencionista e fragmentado, com pouco impacto na melhoria das condições de saúde da população. Além disso, como salientam Giovanella e Mendonça (2012), também ganhavam destaque as discrepâncias de acesso aos serviços de saúde entre os países desenvolvidos e em desenvolvimento.

Entretanto, para Mendes (2002), as discussões sobre a APS já podiam ser observadas na Europa décadas antes. De acordo com o autor, o termo Atenção Primária à Saúde foi empregado em 1920 pelo Relatório Dawson, no Reino Unido, para designar a ideia de instauração de uma porta de entrada organizadora dos sistemas universais de saúde. Elaborado para responder à solicitação do MS inglês no esforço de reconstrução do país após a Primeira Guerra Mundial, esse relatório sugeria a criação de um sistema de saúde público que conjugasse ações de caráter preventivo e curativo, desenvolvidas 
ATENÇÃO PRIMÁRIA À SAÚDE NA AGENDA PÚBLICA BRASILEIRA:

dilemas entre focalização e universalidade

por profissionais generalistas de forma integrada a especialistas e organizadas a partir de três eixos principais: os centros primários de atenção à saúde; os centros secundários e os hospitais-escola, além dos serviços domiciliares.

As proposições contidas no Relatório Dawson receberam, contudo, muitas críticas e resistências e não foram imediatamente acatadas naquele momento. Porém, grande parte das propostas contidas nesse documento foi encampada no processo de institucionalização do National Health Services (NHS) britânico, em 1948, favorecido pela conjuntura do segundo pós-guerra que instituiu o Welfare State. Nos anos de 1960, foram expandidos muitos centros de saúde na Inglaterra, onde os médicos generalistas passaram a atuar juntamente com outros profissionais. (GIOVANELLA; MENDONÇA, 2012).

Nesses termos, os serviços de enfermagem domiciliar, de saúde coletiva, de promoção e de prevenção de saúde passaram a ganhar visibilidade crescente no sistema de saúde inglês e faziam-se presentes em assuntos de habitação, vias públicas e higiene. Estavam, assim, lançadas duas características básicas da APS que influenciariam a organização dos sistemas de saúde em todo o mundo: a regionalização, ancorada na ideia de que os serviços sanitários devem estar organizados com base na população e nas necessidades de saúde; e a integralidade, pautada na perspectiva de indissociabilidade entre ações preventivas e curativas. (FAUSTO; MATTA, 2007).

A partir da experiência inglesa, um amplo debate em favor da medicina preventiva foi ganhando espaço em diferentes países, inspirando iniciativas bastante diversas de cuidados primários, especialmente nos anos 1960 e 1970. Destacam-se aqui as experiências dos Estados Unidos, que na década de 1960 apostavam na medicina familiar e comunitária como eixos relevantes no combate à pobreza, voltadas para programas assistenciais e a do Canadá, que publicou em 1974 o relatório Lalonde, enfatizando a promoção da saúde e a relação da eficácia da medicina com condições de vida da população, aí incluindo estilo de vida, condições biológicas, ambientais e sociais e a eficiência dos serviços de saúde oferecidos. (AGUIAR, 2003).

Essas experiências relacionadas à Atenção Primária fomentaram os debates realizados na Conferência Internacional sobre Cuidados Primários em Saúde de Alma-Ata. O evento contou com o apoio 
da Organização Mundial de Saúde (OMS) e do Fundo das Nações Unidas para Infância (UNICEF) e teve a participação de delegações de 134 países. Essa Conferência é considerada um marco referencial para a APS no mundo, haja vista que declarou a saúde como um direito humano fundamental e enfatizou a importância da APS na organização dos sistemas de saúde. Além disso, segundo Aguiar (2003), o relatório final do referido evento definiu e enfatizou o conceito de APS e seus princípios, a partir da avaliação e proposição de estratégias de superação dos problemas sanitários, definindo ainda as responsabilidades dos governos em relação ao desenvolvimento da APS no âmbito internacional.

\section{A Conferência de Alma-Ata definiu a APS como}

[...] cuidados essenciais baseados em métodos de trabalho e tecnologias de natureza prática, cientificamente críveis e socialmente aceitáveis, universalmente acessíveis na comunidade aos indivíduos e às famílias, com a sua total participação e a um custo suportável para as comunidades e para os países, à medida que se desenvolvem num espírito de autonomia e autodeterminação. (MENDES, 2012, p. 56).

Foram ainda definidos oito elementos essenciais que compõem o nível de Atenção Primária à Saúde: a educação em saúde, a promoção da alimentação saudável, o saneamento básico, o planejamento familiar, imunização contra doenças infecciosas, a prevenção de endemias, a provisão de medicamentos e o tratamento adequado para as doenças mais comuns. A atenção ambulatorial de primeiro nível, isto é, os serviços de primeiro contato do paciente com o sistema de saúde, direcionados a cobrir as afecções e condições mais comuns, e resolver a maioria dos problemas de saúde de uma população são em geral denominados de serviços de atenção primária. Além disso, a APS foi apontada como estratégia para expansão dos sistemas nacionais de saúde, de forma a alcançar, assim, a meta do movimento Saúde Para Todos até o ano 2000.

A definição tanto do conceito de APS quanto da diretriz de saúde para todos apresenta certa flexibilidade, de acordo com a estrutura de cada país, na medida em que busca levar em consideração as características socioeconômicas e as condições de vida e de saúde dos diferentes segmentos populacionais. Nesses termos, é preciso reconhecer que a implementação da APS não seguiu um padrão único em todos os países, assim como não há uniformidade na interpre- 
ATENÇÃO PRIMÁRIA À SAÚDE NA AGENDA PÚBLICA BRASILEIRA:

dilemas entre focalização e universalidade

tação do termo Atenção Primária à Saúde, empregado para referir-se a diferentes concepções.

Analisando experiências de APS em diferentes países, Mendes (2002) identificou diferentes abordagens, por ele agrupadas em três eixos principais: a atenção primária à saúde com caráter seletivo, como nível primário e como estratégia de organização do sistema de serviços de saúde.

A interpretação da atenção primária à saúde com caráter seletivo entende-a como um programa focalizado na população e regiões pobres, oferecendo tecnologia simples de baixo custo. A interpretação que inscreve como nível primário do sistema caracteriza-se como porta de entrada na rede de saúde, com ações de atenção de primeiro nível. E a terceira interpretação inscreve-se como estratégia de organização do sistema de serviços de saúde, entendida como forma de reorganizar e reordenar todos os recursos que o sistema dispõe para satisfazer as demandas da população. Isso implica conceber a atenção primária à saúde a função de articulador dentro de um sistema integrado de saúde.

As formas de organização da atenção primária também apresentam variações, agregadas no cenário contemporâneo em duas alternativas: os sistemas fragmentados e os sistemas integrados. (MENDES, 2002). Os sistemas fragmentados apontam para uma (des) organização do sistema através de conjuntos de pontos de serviços de saúde isolados, assistemáticos e incomunicáveis entre si, fragilizando uma continuidade dos serviços prestados. Porém, os sistemas integrados de serviços de saúde, mediados e organizados pela atenção primária, incorporam uma rede integral de pontos de atenção à saúde que oferecem uma assistência sistematizada e contínua a uma população definida.

Apesar da importância de Alma-Ata, o contexto que se seguiu a sua realização foi pautado pela disseminação, no plano internacional, de uma concepção restrita e seletiva de APS. Propagada especialmente pelo Banco Mundial (BM), essa concepção baseava-se na formulação de programas com o objetivo de reduzir problemas específicos de saúde, dirigidos a grupos populacionais específicos em situação de pobreza. Propunha, para isso, o uso de recursos de baixa densidade tecnológica, sem articulação com outros recursos em saúde, limitando-se a ações de imunização, estímulo ao aleitamento materno, acompanhamento do crescimento e desenvolvimento das 
crianças, reidratação oral, suplementação alimentar e planejamento familiar. (AGUIAR, 2003). Advogava-se, desse modo, que esses serviços básicos de saúde deveriam ser providos pelo Estado para os segmentos populacionais que não tivessem condições de arcar com os gastos em saúde, reforçando o caráter segmentado da atenção à saúde.

Em contraposição a essa tendência, alguns países desenvolveram propostas que buscaram valorizar estratégias participativas e intersetoriais como forma de garantir o direito universal à saúde. Trata-se de iniciativas como as Cidades Saudáveis, no Canadá, e os Sistemas Locais de Saúde (SILOS), difundidos pela OMS. (FAUSTO, 2005). Essa autora avalia, entretanto, que, em geral, essas iniciativas pouco avançaram em termos concretos, na medida em que o foco central permaneceu na ação intersetorial a partir dos serviços de saúde e nas ações curativas.

Recentemente observa-se um movimento de renovação da atenção primária impulsionado pelas agências internacionais de saúde - OMS e Organização Pan-Americana da Saúde (OPAS) - como forma para alcançar os Objetivos do Milênio acordados pelas Nações Unidas. Em 2003, a OPAS/OMS aprovou resolução que insta os Estados Membros a adotarem uma série de recomendações para fortalecer a APS e em 2005 divulgou o documento de posicionamento Renovação da Atenção Primária em Saúde nas Américas. Esse documento defende a necessidade de se alcançar atenção universal e abrangente, por meio de uma abordagem integrada e horizontal para o desenvolvimento dos sistemas de saúde, com cuidados orientados à qualidade, ênfase na promoção e prevenção, intersetorialidade, participação social, e responsabilização dos governos. (ORGANIZAÇÃO PAN-AMERICANA DE SAÚDE, 2005).

Seguindo essa tendência, em 2008, no esteio das comemorações pelos trinta anos da Declaração de Alma-Ata, a OMS lançou o Relatório Mundial da Saúde, com o título Atenção Primária à Saúde: agora mais do que nunca. De acordo com Giovanella e Mendonça (2012), esse relatório enfatizou a renovação para o desenvolvimento da APS, considerando-a não apenas como um programa focalizado para a população empobrecida, mas que abarca elementos que buscam garantir o princípio da universalidade, do atendimento às demandas coletivas e inscreve a participação social com importante papel nesse processo. Para Silveira, Machado e Mata (2015), a re- 
ATENÇÃO PRIMÁRIA À SAÚDE NA AGENDA PÚBLICA BRASILEIRA:

dilemas entre focalização e universalidade

novação no conceito da APS reforça a universalidade e questiona a focalização da política, enfatiza ações de caráter democrático e coletivo e destaca, também, a intersetorialidade, como elemento essencial que inscreve esse nível de atenção como alicerce da organização do sistema público de saúde.

A renovação proposta pela OMS subentende, portanto, uma atualização da concepção abrangente de APS. Sua implementação, contudo, dependerá da tradução dos princípios enunciados em práticas concretas, assim como da adesão dos diferentes governos nacionais.

Como visto, o debate internacional da atenção primária ganha destaque em 1978, conjuntura de profunda crise econômica do capitalismo mundial que introduz novas formas de gestão da economia, propostas, principalmente, pelos organismos internacionais, como o BM. Aguiar (2003) ressalta que a revisão dos rumos para as políticas sociais, a racionalização dos investimentos no público, o aumento da pobreza e a autonomia do mercado trouxeram impactos para a implementação da APS no mundo e o Brasil não se encontra isento dessas tendências e vivencia tensões entre o campo teórico e prático da implementação da Atenção Primária à Saúde.

3 ATENÇÃO PRIMÁRIA À SAÚDE NO BRASIL: avanços, contradições e desafios

No Brasil, a Atenção Primária à Saúde ganha destaque na agenda setorial a partir de meados dos anos 1990, com a implantação da ESF. Entretanto, estudos como os de Mendes (2002), Aguiar (2003) e Fausto (2005) identificam que experiências relacionadas a esse campo tiveram espaço em diferentes momentos da trajetória histórica do país.

De fato, a emergência da organização das ações e serviços de saúde no Brasil se dá ainda na Primeira República (1891-1930), com ênfase na saúde coletiva e no combate às endemias. De acordo com Lima (2002), o movimento médico sanitário que surgiu naquele contexto desempenhou um papel importante na consolidação do Estado brasileiro, na medida em que as concepções sobre o processo saúde-doença e o modo de lidar com ele tinha implicações para a economia, o comércio internacional e o desenvolvimento científico e tecnológico do país. 
Ainda no período, foram implantados postos de saneamento e profilaxia rural, que subsidiaram a criação dos centros de saúde. A ação desses centros de saúde ancorava-se em um modelo de cunho educativo e preventivo, com o objetivo de estimular uma consciência sanitária na população, especialmente a mais pobre. (CAMPOS, 2006). Contando com a atuação de enfermeiras visitadoras e sob forte influência higienista, os centros de saúde buscavam conhecer a situação dos domicílios, famílias e indivíduos, de forma a estabelecer o controle sanitário e ensinar hábitos e comportamentos saudáveis.

A partir da década de 1930, com o reconhecimento da questão social, a saúde, a exemplo de outras áreas sociais, passou a ser função estatal, estabelecendo um modelo dual que perduraria pelo menos até os anos 1980: de um lado, a saúde pública, de acesso universal e com ações voltadas para o controle das epidemias e, de outro lado, a medicina previdenciária, de caráter individual e curativo, que fazia da carteira de trabalho o certificado de direito para acesso à política pública de saúde. (SIMIONATTO, 1997). Gradativamente, a medicina previdenciária tornou-se central no modelo de intervenção do Estado brasileiro na saúde, encampando a quase totalidade dos recursos financeiros públicos do setor e reforçando a dualidade da política nacional de saúde. Durante o período da Ditadura Militar (1964-1985), a medicina previdenciária expande-se e consolida-se, à custa principalmente da contratação de serviços privados, por meio de convênios e credenciamentos. (GIOVANELLA; MENDONÇA, 2012).

A partir de meados dos anos 1970, o modelo médico-previdenciário entra em crise, decorrente não apenas do modelo de expansão da oferta de serviços de saúde adotado, mas também do aprofundamento da crise econômica do capitalismo mundial. Esse contexto favoreceu o debate crítico em torno do modelo de atenção à saúde e permitiu a proliferação de experiências sanitárias locais baseadas na medicina comunitária, muitas delas vinculadas aos Departamentos de Medicina Preventiva das universidades públicas. (FAUSTO, 2005).

Ao mesmo tempo, o governo federal adotou algumas iniciativas de expansão de cobertura assistencial, com foco nas populações marginalizadas e desprovidas de acesso aos serviços sociais. Destaca-se, nesse sentido, a elaboração, em 1976, do Programa de Interiorização das Ações de Saúde e Saneamento (PIASS), que designava 
ATENÇÃO PRIMÁRIA À SAÚDE NA AGENDA PÚBLICA BRASILEIRA:

dilemas entre focalização e universalidade

recursos para a construção de Unidades Básicas de Saúde, principalmente nas cidades menores do Nordeste brasileiro e a formulação do Programa Nacional de Serviços Básicos de Saúde (PREVSAUDE), em 1981. Esse último proponha uma reforma no sistema nacional de saúde a partir da universalização dos cuidados primários. No entanto, não logrou êxito, tendo enfrentado fortes resistências de grande parte da burocracia estatal, do segmento médico-empresarial e do campo da medicina liberal. (FAUSTO, 2005).

Em 1985, já no primeiro ano de governo da Nova República, ganha expressão o programa de Ações Integradas de Saúde (AIS). As AIS propunham a articulação entre as três esferas governamentais na provisão e gestão das ações de saúde, pautando-se pelos princípios de integração interinstitucional, descentralização e democratização. Estabeleciam a organização do primeiro nível de atenção por meio das unidades básicas municipais, que deveriam prestar atenção integral a toda a população, independente da contribuição financeira prévia. Com as AIS, houve significativa expansão da rede básica de saúde, que passou a oferecer, além das atividades preventivas, atendimento médico em clínica, pediatria e gineco-obstetrícia. (GIOVANELLA; MENDONÇA, 2012).

A VIII Conferência Nacional de Saúde (CNS), realizada em 1986, foi um marco importante na direção da construção do SUS. Pela primeira vez, uma conferência de saúde contou com ampla participação social, através de representantes de movimentos sociais e profissionais de saúde. O relatório final dessa CNS estabeleceu a unificação do sistema de saúde, com definição do conceito ampliado do processo saúde-doença e das bases financeiras do sistema, lançando, assim, a pedra fundamental do SUS. Fausto (2005) menciona que a VIII CNS não enfatizou a APS como estratégia de reorientação da atenção à saúde, na medida em que naquele momento foram priorizados os esforços para garantir uma proposta mais ampla de reformulação do sistema de saúde.

Em 1987, as AIS são substituídas pelos Sistemas Unificados e Descentralizados de Saúde (SUDS), com o objetivo de contribuir para a conformação de um sistema descentralizado e com comando único. Para Fausto (2005), no contexto do SUDS a questão do modelo de atenção à saúde ficou em segundo plano.

A CF de 1988 inscreveu a saúde no âmbito da seguridade social brasileira, ao lado da previdência e da assistência social, abrin- 
do, assim, a perspectiva de reconhecê-la como direito de todos e dever do Estado. Como destaca Lobato (2012), a noção de seguridade social trouxe avanços na institucionalização de um modelo ampliado de proteção social, que previa a universalização do acesso, a responsabilidade estatal, a integração entre as três áreas e a criação de um orçamento próprio, exclusivo e com novas fontes de financiamento. Além disso, o texto constitucional instituiu, de forma inédita, a responsabilidade compartilhada entre os três níveis de governo na gestão e provisão da atenção à saúde, através da descentralização das ações e recursos e na participação e controle social, por meio da implantação de mecanismos colegiados de deliberação, tais como os conselhos de políticas públicas.

Lobato (2012) analisa que os marcos constitucionais significaram uma ruptura com o formato de intervenção do Estado na questão social, ao menos do ponto de vista normativo-legal. Mas chama atenção, ao mesmo tempo, para os constrangimentos postos pelas fragilidades da própria estrutura social brasileira naquele momento, caracterizada por baixos níveis de organização, pelo predomínio do autoritarismo nas relações sociais e institucionais e pelas profundas desigualdades sociais.

No caso específico da saúde, a criação do SUS promoveu o campo sanitário à condição de primeira política social universal no Brasil, resultando na ampliação do acesso a milhares de brasileiros às ações de saúde. Mas, ao mesmo tempo, sua institucionalização tal como inscrito no texto constitucional tem sido atravessada por importantes dilemas e conflitos, em grande parte derivados do trade-off entre a consolidação de uma institucionalidade democrática e a adoção da agenda neoliberal pelos sucessivos governos que se seguiram.

É no interior dessa tensão que a APS ganha posição de destaque na agenda nacional, inicialmente com a criação, em 1991, do Programa de Agentes Comunitários em Saúde (PACS) e, posteriormente, em dezembro de 1993, do Programa Saúde da Família (PSF). A perspectiva de investimentos na atenção básica é, como visto anteriormente, uma ideia cara às propostas da reforma sanitária brasileira, emergindo da crítica ao modelo médico hegemônico, focado na cura e na atenção especializada.

Contudo, a implantação do PACS e do PSF sofreu severas críticas por estar supostamente atualizando modelos focalizados e 
seletivos de atenção à saúde. Essas críticas procediam, à medida que tais programas foram implantados com a perspectiva de expandir a cobertura assistencial a regiões pauperizadas e desprovidas de serviços. Além disso, esses programas eram marginais à estrutura de serviços, sem financiamento próprio e imersos no contexto de redução dos investimentos em saúde. (GIOVANELLA; MENDONÇA, 2012).

A par dos seus limites, autores como Castro e Fausto (2012) identificam nesses programas avanços quanto à introdução de novas concepções na organização do modelo de atenção à saúde, em especial quanto ao reconhecimento das necessidades em saúde como um processo social. Além disso, progressivamente, a Saúde da Família vai perdendo seu caráter de programa e passa a assumir a configuração de estratégia prioritária para reorientação do modelo assistencial em saúde, inclusive mudando sua denominação para ESF.

Essas autoras destacam um conjunto de medidas adotadas pelo governo federal para ampliar a perspectiva de APS, muitas delas decorrentes da implantação e desenvolvimento da própria ESF. Em primeiro lugar, cabe sublinhar as mudanças nos mecanismos de financiamento da saúde introduzidas a partir da Norma Operacional Básica de 1996 (NOB-96). Essa NOB estabeleceu financiamento próprio para as ações básicas em saúde, criando um mecanismo então inédito de repasse de recursos com base per capita, dirigidos ao desenvolvimento de ações e serviços no âmbito da APS, além de incentivos financeiros para que os municípios implantassem equipes de Saúde da Família. Tais alterações contribuíram para uma importante expansão da ESF, antes restrita à adesão pontual.

Em 1999, foi criado, em âmbito ministerial, o Departamento Nacional de Atenção Básica (DAB), voltado à gestão nacional da ESF. Em 2000, esse departamento passou a ser subordinado à Secretaria de Políticas de Saúde (SPS), em uma tentativa de articulação com outras políticas do Ministério da Saúde, de forma a ultrapassar seu caráter vertical e paralelo às demais intervenções.

A edição das Normas Operacionais de Atenção à Saúde (NOAS) pelo Governo Federal em 2001/2002 buscou reforçar a APS como central na política nacional de saúde, propondo ações para melhorar e qualificar a resolutividade da APS em todos os municípios, a partir da identificação de áreas estratégicas de atuação. Essas normativas tinham como foco central a constituição de meca- 
nismos de indução à construção de sistemas regionalizados e integrados de serviços de saúde.

Em 2003, outra medida adotada pelo Ministério da Saúde para ampliação da ESF foi o Projeto de Expansão e Consolidação da Saúde da Família (PROESF), voltado para a organização e o fortalecimento da APS nos grandes centros urbanos, localidades onde a cobertura da ESF tende a ser muito baixa. De acordo com avaliações do próprio MS (BRASIL, 2008), o PROESF contribuiu para o fortalecimento da APS no país, através do aumento da cobertura da ESF e aumento do número de municípios com equipes implantadas.

A ESF também fomentou a elaboração da Política Nacional de Atenção Básica (PNAB), em 2006, a qual contribuiu para reforçar uma concepção abrangente de APS, que valorizava tanto a dimensão preventiva quanto a clínica. (CASTRO; FAUSTO, 2012). A PNAB sofreu revisão no ano de 2011, quando uma nova versão dessa política foi editada, estabelecendo, entre outras alterações, a possibilidade de conformação de equipes parametrizadas, ou seja, com composição diferente da originalmente instituída. Em 2008, foram criados os Núcleos de Apoio à Saúde da Família (NASF), que incorporam outras categorias profissionais para atuarem em parceria com as equipes de SF seguindo a lógica de apoio matricial baseado na regionalização da saúde. E no ano de 2011, o MS implantou o Programa de Melhoria do Acesso e da Qualidade da Atenção Básica (PMAQ-AB), que instituiu um componente de qualidade vinculado ao desempenho das equipes de Saúde da Família no financiamento setorial.

A essas iniciativas se somam outras ações, de diferentes modalidades, levadas a cabo pelo Ministério da Saúde para fomentar mudanças no modelo de atenção à saúde. Tais ações incluem os processos de formação e qualificação dos profissionais de saúde, a interiorização dos programas de saúde e fixação de profissionais, principalmente médicos, nas equipes de ESF.

O alcance da ESF é significativo: em dezembro de 2014, o Brasil contava com 47.810 equipes credenciadas pelo Ministério da Saúde, distribuídas por 5.465 municípios $(98,3 \%)$ e com cobertura populacional estimada em $62,5 \%$, o que corresponde a mais de 121 milhões de brasileiros. (POPULAÇÃO..., 2015). Esses números indicam indubitáveis avanços no que diz respeito à expansão da APS no país. No entanto, registram-se também desafios ainda não supe- 
ATENÇÃO PRIMÁRIA À SAÚDE NA AGENDA PÚBLICA BRASILEIRA:

dilemas entre focalização e universalidade

rados, especialmente no que tange a sua consolidação dentro de uma lógica universal e equitativa. Esses desafios são ainda maiores frente à persistência de uma política econômica voltada à priorização de medidas de ajuste estrutural, que apontam em direção à adoção de políticas sociais segmentadas, fragmentadas e focalizadas nos setores mais pobres. No caso da ESF, observam-se conflitos entre diferentes concepções de APS, opondo perspectivas seletivas e abrangentes, as quais compreendem arcabouços teóricos, ideológicos e metodológicos diferentes, com implicações também distintas para o direito universal à saúde.

Em termos gerais, a ESF tem encontrado dificuldades para incorporar os estratos médios da população, como demonstra o trabalho de Davies (2015) sobre uma experiência local na cidade do Rio de Janeiro, limitando-se à camada mais empobrecida da população. Corrobora essa argumentação o estudo realizado por Bousquat, Cohn e Elias (2006) sobre a implantação ESF no município de São Paulo, a partir de um enfoque sobre o espaço urbano. Os autores identificaram que a implantação da ESF se distribui de forma heterogênea entre as diversas regiões da cidade, mostrando-se consolidada apenas nos estratos de maior exclusão social, ao passo que os estratos superiores apresentam a menor cobertura da ESF.

Em municípios de pequeno porte e distantes dos grandes centros urbanos, dificuldades relacionadas à fixação de profissionais, sobretudo médicos, e à integração com outros níveis do sistema de saúde são referidos na literatura. (GIOVANELLA; MENDONÇA, 2012), ainda que indicadores de provisão apontem para uma considerável universalização da ESF em pequenas e médias cidades. (COSTA, 2016).

Indicativo da tendência de segmentação do SUS está na composição relativa dos usuários do sistema de serviços de saúde no país. Informações disponibilizadas pelo Conselho Nacional de Secretários de Saúde (CONASS, 2006) a partir de uma pesquisa de opinião realizada no ano de 2003 , apontavam que $28,6 \%$ dos brasileiros eram usuários exclusivos do SUS, $61,5 \%$ eram usuários não exclusivos e apenas $8,7 \%$ não utilizavam o SUS. Essa distribuição variava entre as regiões geográficas do país, porte dos municípios e localização urbano ou rural, mas em todas essas variações, o percentual dos que eram usuários não exclusivos era maioria. Esse quadro caracteriza uma "[...] mobilidade unilateral da demanda." (CON- 
SELHO NACIONAL DE SECRETÁRIOS DE SAÚDE, 2006, p. 58), em que a população coberta pelo sistema de saúde suplementar utiliza, com frequência, os serviços públicos de maior densidade tecnológica e, portanto, de alto custo, enquanto aqueles não cobertos pelos planos privados de saúde não podem utilizar o serviço privado, configurando o que Médici (2005) denomina de seleção adversa no SUS. Desse modo, o SUS caminha como parte de um sistema segmentado, ao lado do sistema de saúde suplementar e do desembolso direto.

Essa tendência é ainda enfatizada quando se examina o perfil dos gastos em saúde no Brasil e em outros países que adotam sistemas universais de saúde. Como pode ser observado na Tabela 1, a participação do gasto total em saúde em relação ao Produto Interno Bruto (PIB) no Brasil era, em 2011, próxima a dos países com sistemas universais públicos de saúde, tais como Suécia e Reino Unido. No entanto, quando se examina o gasto per capita, verifica-se a discrepância em relação a esses mesmos países, o que indica que, em termos internacionais, o Brasil gasta pouco em saúde.

Tabela 1 - Gastos em saúde no Brasil e em países selecionados, 2011

\begin{tabular}{l|c|c|c}
\hline \multicolumn{1}{c|}{ Países } & $\begin{array}{c}\text { Gasto total em saúde } \\
\text { como } \% \text { do PIB }\end{array}$ & $\begin{array}{c}\text { Gasto per capita total } \\
\text { em saúde (US\$) }\end{array}$ & $\begin{array}{c}\text { Gasto governamental em } \\
\text { saúde como \% do PIB }\end{array}$ \\
\hline CUBA & 8,6 & 558 & 94,2 \\
\hline REINO UNIDO & 9,3 & 3595 & 84,0 \\
\hline SUÉCIA & 9,6 & 5293 & 81,3 \\
\hline ALEMANHA & 11,3 & 4717 & 76,7 \\
\hline CANADA & 10,9 & 5763 & 69,3 \\
\hline ARGENTINA & 6,8 & 994 & 47,5 \\
\hline BRASIL & 9,5 & 1078 & 47,0 \\
\hline ESTADOS UNIDOS & 17,0 & 8845 & 2014. Dispo \\
\hline
\end{tabular}

Fonte: WORLD HEALTH ORGANIZATION. World Health Statistics 2014. Switzerlan, 2014. Disponível em:<http://apps.who.int/iris/bitstream/10665/112738/1/9789240692671_eng.pdf>. Acesso em: 10 jan. 2016.

Além disso, dentre os países selecionados, o Brasil é o que apresenta um dos menores percentuais $(47,5 \%)$ de gastos públicos em saúde em relação ao PIB, superando apenas os Estados Unidos. Não é exagero afirmar que essa estrutura de gastos em saúde se mostra limitada, para não dizer incompatível, com a perspectiva de construção de um sistema público universal de qualidade.

Outra questão que merece destaque é a articulação da ESF com os outros níveis do sistema de saúde (média e alta complexidades). Entende-se que essa articulação remete ao conceito de integra- 
ATENÇÃO PRIMÁRIA À SAÚDE NA AGENDA PÚBLICA BRASILEIRA:

dilemas entre focalização e universalidade

lidade da atenção, definida como a garantia do direito de acesso dos usuários às ações e serviços dos diferentes níveis de complexidade, indispensáveis para atendimento das necessidades de saúde. Desse modo, abre-se a possibilidade de romper com a perspectiva de APS como uma cesta básica de serviços de saúde dirigidas aos mais pobres.

No entanto, a integração entre os diferentes níveis de complexidade segue como importante desafio. Persistem importantes barreiras de acesso a exames e consultas especializadas, o que afeta a continuidade da assistência prestada no âmbito da ESF, um dos atributos centrais de uma concepção de APS abrangente. Conforme argumenta Bravo (2006, p. 4),

\begin{abstract}
O programa [Saúde da Família] precisa ter sua direção modificada na perspectiva de prover atenção básica em saúde para toda a população de acordo com o princípio da universalidade. Para garantir a integralidade, o programa precisa ter como meta a (re)organização do sistema como um todo, prevendo a articulação da atenção básica com os demais níveis de assistência.
\end{abstract}

Essa mesma autora destaca outra questão, também fundamental na conformação da APS, que é a contratação de recursos humanos para atuar na ESF. Essa questão exemplifica a hostilidade da lógica neoliberal sobre a política de saúde, uma vez que a precarização das condições de trabalho, via terceirização com ausência de concurso público, inviabiliza o compromisso com os princípios norteadores do SUS, gerando rotatividade dos profissionais e afetando a construção de vínculos entre as equipes e entre essas e a população adscrita. A exemplo dos achados de Marqui e outros (2010), ao analisarem a conformação e o processo de trabalho de equipes da ESF em uma localidade do Rio Grande do Sul, os profissionais que atuam na ESF tendem a ser predominantemente jovens e com formação profissional recente, com vínculos de trabalho precário, o que demonstra as dificuldades da APS em atrair profissionais em desenvolvimento de carreira, sobretudo os médicos.

Esse conjunto de questões aponta para a persistência de um modelo segmentado de atenção à saúde, a despeito dos diversos avanços conquistados ao longo das quase três décadas de construção do SUS e do recente fortalecimento da APS como estratégia de reorganização da atenção à saúde. A defesa de sistemas segmentados passa pelo argumento de que a instituição de sistemas especiais para 
aqueles que podem pagar permite destinar os recursos sobrantes para $\mathrm{o}$ atendimento aos pobres. No entanto, evidências empíricas apontam exatamente o contrário, na medida em que a instituição exclusiva de um sistema para pobres leva a um subfinanciamento e amplia as iniquidades em saúde. (CONSELHO NACIONAL DE SECRETÁRIOS DE SAÚDE, 2006).

Nesse cenário antagônico entre focalização e universalização das práticas da Atenção Primária no país, há possibilidades de superar a implementação de uma política focada na população pobre, pois se observa que a APS é um dos espaços institucionais que viabiliza o contato direto aos usuários, favorecendo o desenvolvimento de práticas inovadoras, possibilidades de mudanças e novas perspectivas para o profissional e para o usuário da saúde.

Entretanto, a experiência tem mostrado que não basta a adoção de mudanças no âmbito da APS. A efetiva construção do SUS em direção a um sistema público, universal, equânime e de qualidade requer enfrentar várias frentes simultaneamente.

Nessa perspectiva, a atenção básica em saúde apresenta potencialidades na direção de viabilizar a construção de espaços coletivos de estímulo à participação dos usuários no espaço público. Acredita-se que esse processo contribuiria para a democratização de informações e a produção do conhecimento que estimule o senso crítico na perspectiva da construção da cidadania.

\section{CONCLUSÃO}

Verifica-se que a Atenção Primária à Saúde ganha destaque no Brasil, impulsionada pelos dilemas entre o processo de consolidação democrática e o contexto de mudanças macrossocietárias postas pela emergência de políticas neoliberais e pela consolidação do processo de reestruturação produtiva que estabelece um quadro recessivo às políticas sociais. Nesse contexto, a universalidade da APS se torna alvo de críticas pelos principais organismos internacionais, que defendem os propósitos dos grandes capitalistas e designam com caráter seletivo e focalizado na implementação das APS no país.

Sob a égide neoliberal, um risco importante é o de que a Atenção Primária à Saúde se consolide como uma política restrita aos segmentos mais pobres da nossa população, compatível com as estratégias do denominado universalismo básico. (MINTEGUIAGA, 
ATENÇÃO PRIMÁRIA À SAÚDE NA AGENDA PÚBLICA BRASILEIRA:

dilemas entre focalização e universalidade

2009). Nessa perspectiva, tende a predominar o caráter seletivo da ESF, constituindo uma espécie de cesta básica de procedimentos de baixo custo dirigidos ao conjunto da população, ao passo que nos procedimentos mais complexos e de maior custo prevalece, de forma bastante intensa, a lógica mercantil, com forte expansão do mercado de planos privados de saúde nos estratos de renda média e alta.

Se é possível, de um lado, afirmar que houve um esforço importante de expansão da oferta de serviços de saúde da APS nas últimas décadas, de outro, observa-se que essa expansão se deu de forma desigual e heterogênea no território nacional. As recentes iniciativas de constituição das Redes de Atenção à Saúde confirmam o ainda frágil desempenho da APS como eixo organizador da rede de serviços de saúde e política universal em conformidade com os princípios norteadores do SUS.

Tal reconhecimento exige recuperar uma perspectiva crítica de análise sobre os constrangimentos que se impõem ao SUS como direito de cidadania, ao mesmo tempo em que requer a construção de estratégias que fortaleçam, ampliem e ampliem a noção de direito universal sob responsabilidade pública.

\section{REFERÊNCIAS}

AGUIAR, R. A. T. A Construção Internacional do Conceito de Atenção Primária à Saúde (APS) e sua influência na emergência e consolidação do Sistema Único de Saúde no Brasil. 2003. 156 f. Tese (Doutorado) - Faculdade de Medicina, Universidade Federal de Minas Gerais, Belo Horizonte, 2003.

BAPTISTA, T. W. F. O direito à saúde no Brasil: sobre como chegamos ao Sistema Único de Saúde e o que esperamos dele. In: Escola Politécnica de Saúde Joaquim Venâncio (Org.) Textos de Apoio em Políticas de Saúde. Rio de Janeiro: FIOCRUZ, 2005.

BOSCHETTI, I. Implicações da Reforma da Previdência na Seguridade Social Brasileira. Revista Psicologia e Sociedade, Belo Horizonte, v. 15, n. 1, p. 57-96, jan./jun. 2003.

BOUSQUAT, A.; COHN, A.; ELIAS, P. E. Implementação do Programa Saúde da Família e exclusão sócio-espacial do município de São Paulo, Brasil. Cadernos de Saúde Pública, Rio de Janeiro, v. 22, n. 9, p. 1935-1943, set. 2006. 
BRASIL, Ministério da Saúde. Secretaria de Atenção à Saúde. Departamento de Atenção Básica. Portaria n ${ }^{\circ}$ 154, de 24 de janeiro de 2008. Cria os Núcleos de Apoio à Saúde da Família - NASF. Diário Oficial da União, Brasília, DF, 2008.

BRAVO, M. I. Serviço Social e Reforma Sanitária: lutas sociais e práticas profissionais. São Paulo: Cortez, 2006.

CAMPOS, C. E. A. A organização dos serviços de Atenção Primária à Saúde no Brasil. Revista Brasileira de Medicina de Família e Comunidade, Rio de Janeiro, v. 2, n. 6, p. 131-147, 2006.

CASTRO, A. L. B.; FAUSTO, M. C. R. A Política Brasileira de Atenção Primária à Saúde. In: MACHADO, C. V. (Org.). Políticas de Saúde no Brasil: continuidade e avanços. Rio de Janeiro: FIOCRUZ, 2012. p. 173-194.

CONSELHO NACIONAL DE SECRETÁRIOS DE SAÚDE. SUS: avanços e desafios. Brasília, DF, 2006.

COSTA, N. do R. A Estratégia de Saúde da Família, a atenção primária e o desafio das metrópoles brasileiras. Ciência e Saúde Coletiva, Rio de Janeiro, v. 21, n. 5, p. 1389-1398, 2016. Disponível em $<$ http://www.scielo.br/scielo.php?script=sci_arttext\&pid=S1413$81232016000501389 \& \operatorname{lng}=$ en\&nrm=iso $>$. Acesso em: 20 jul. 2016.

DAVIES, W. H. O acesso da classe média à Estratégia de Saúde da Família em contexto de expansão da Atenção Primária à Saúde no município do Rio de Janeiro. 2015. Dissertação (Mestrado) - Escola Nacional de Saúde Pública, Fundação Oswaldo Cruz, Rio de Janeiro, 2015.

FAUSTO, M. C. R. Dos programas de medicina comunitária ao Sistema Único de Saúde: uma análise histórica da atenção primária na política de saúde brasileira. 2005. Tese (Doutorado) - Instituto de Medicina Social, Universidade do Estado do Rio de Janeiro, Rio de Janeiro, 2005.

; MATTA, G. Atenção Primária à Saúde: histórico e perspectivas. In: MOROSINI, V. G. C.; CORBO, A. D. (Orgs.). Modelos de atenção e a saúde da família. Rio de Janeiro: EPSJV/ FIOCRUZ, 2007. p. 43-67.

FRANCO, T. B.; MEHRY, E. Programa de Saúde da Família: contradições de um programa destinado à mudança do modelo tecnoassistencial. [S. 1.: s. n.], 2000. Mimeo. 
ATENÇÃO PRIMÁRIA À SAÚDE NA AGENDA PÚBLICA BRASILEIRA:

dilemas entre focalização e universalidade

GIOVANELLA, L.; MENDONÇA, M. H. M. Atenção Primária à Saúde. In: GIOVANELLA, L. et al. (Org.). Políticas e Sistema de Saúde no Brasil. Rio de Janeiro: FIOCRUZ, 2012.

LIMA, N. T. O Brasil e a Organização Pan-americana de Saúde: uma história em três dimensões. Rio de Janeiro: [s. n.], 2002.

LOBATO, L. Seguridade Social e Saúde. In SENNA, M. C. M.; ZUCCO, L. P.; LIMA, A. B. R. Serviço Social e Saúde Coletiva. Rio de Janeiro: Garamond, 2012.

MARQUI, A. B. et al. Caracterização das Equipes de Saúde da Família e de seu processo de trabalho. Revista da Escola de Enfermagem da USP, São Paulo, v. 44, n. 4, p. 956-961, 2010.

MEDICI, A. Propostas para melhorar a cobertura, a eficiência e a qualidade no setor saúde. In: BACHA, E. L.; SCHWARTZMAN, S. (Orgs.). Brasil: a nova agenda social. Rio de Janeiro: LCT, 2005.

MENDES, E. V. Atenção Primária à Saúde no SUS. Fortaleza: Escola de Saúde Pública, 2002.

O cuidado das Condições Crônicas na Atenção Primária à Saúde: o imperativo da consolidação da Estratégia da Saúde da Família. Brasília, DF: OPAS, 2012.

MENDONCA, C. S. Saúde da Família, agora mais do que nunca! Ciência e Saúde Coletiva [online], Rio de Janeiro, v. 14, suppl. 1, p. 1493-1497, 2009. ISSN 1678-4561. Disponível em:<http://dx.doi. org/10.1590/S1413-81232009000800022>. Acesso em: 10 jan. 2016.

MINTEGUIAGA, A. De la focalización individual a la focalización de los derechos: crítica a la propuesta del Universalismo Básico. In: LOBATO, L. V. C.; FLEURY, S. (Org.). Seguridade Social, Cidadania e Saúde. Rio de Janeiro: CEBES, 2009. p. 47-65.

ORGANIZAÇÃO PAN-AMERICANA DE SAÚDE. Renovação da Atenção Primária à Saúde nas Américas. Washington, D.C.: PAHO, 2005.

POPULAÇÃO teve acesso a 1,4 bi de consultas médicas pelo SUS em um ano. Blog da Saúde, Brasília, DF, 2015. Disponível em:<www. blog.saude.gov.br/35602-populacao-teve-acesso-a-1-4-bi-deconsultas-medicas-pelo-sus-em-um-ano.html>. Acesso em: 16 maio 2017. 
SILVEIRA, A. C. da; MACHADO, C. V.; MATTA, G. C. A atenção primária em saúde na agenda da organização panamericana de saúde nos anos 2000. Trabalho, Educação e Saúde, Rio de Janeiro, v. 13, n. 1, p. 31-44, abr. 2015.

SIMIONATTO, I. Caminhos e descaminhos da política de saúde no Brasil. Revista Inscrita, Brasília, DF, n. 1, p. 15-20, 1997.

WORLD HEALTH ORGANIZATION. World Health Statistics 2014. Switzerlan, 2014. Disponível em: $<$ http://apps.who.int/iris/ bitstream/10665/112738/1/9789240692671_eng.pdf >. Acesso em: 10 jan. 2016. 